\title{
Efecto de la Suplementación de L-Glutamina sobre la Expresión de Genes de la Respuesta Inmune Humoral de la Mucosa Intesti- nal de Crías de Alpacas (Vicugna pacos)
}

\author{
Effect of Orally Administered L-Glutamine on Gene Expression of the Humoral \\ Immune Response of the Intestinal Mucosa of Baby Alpaca (Vicugna pacos) \\ Emanuel Bejarano L. ${ }^{1}$, Alberto Manchego S. ${ }^{1,4}$, Gina Castro S. ${ }^{1}$, Giovanni Pérez G. ${ }^{3}$, \\ Nieves Sandoval C. ${ }^{2}$, Mercy Ramírez V. ${ }^{1}$
}

\section{Resumen}

\begin{abstract}
El presente estudio tuvo como objetivo determinar el efecto de la L-glutamina sobre la expresión de la inmunoglobulina A(IgA) y la citocinas TGF- $\beta$ y las interleucinas, IL-4, IL5, IL-6 e IL-10 en la mucosa del yeyuno de crías de alpaca de 4-7 días de edad. Se trabajó con un grupo de 6 crías que se le administró $3.3 \mathrm{mM}$ de L-glutamina por kg peso vivo, vía oral, y una segunda dosis a los 7 días. A un segundo grupo de 7 crías se le suministró PBS vía oral como control. Los animales fueron sacrificados 3 días después del tratamiento y se tomó una porción de yeyuno de cada cría. Se realizó la extracción de ARN mensajero (ARNm) y la transcripción reversa $(\mathrm{RT})$ y el PCR tiempo real utilizando cebadores específicos de cada gene en estudio. Para la cuantificación relativa de la IgA, TGF- $\beta$ e interleucinas se usó el método $2^{-\Delta \Delta c t}$. El TGF- $\beta$ se expresó 3 veces más y la IgA 18 veces más en las crías tratadas con L-glutamina que en los controles ( $\mathrm{p}<0.05$ ). Las expresiones de IL-4, IL-5, IL6 e IL-10 tuvieron estadísticamente los mismos niveles en ambos grupos. Se concluye que la L-glutamina incrementa la expresión TGF- $\beta$ e IgA, mejorando la respuesta inmune adaptativa humoral de mucosa intestinal en crías de alpaca.
\end{abstract}

Palabras clave: camélidos sudamericanos, inmunidad de mucosas, glutamina, interleucinas

${ }^{1}$ Laboratorio de Microbiología y Parasitología Veterinaria, ${ }^{2}$ Laboratorio de Histología, Embriología y Patología Animal, Facultad de Medicina Veterinaria, Universidad Nacional Mayor de San Marcos, Lima, Perú

${ }^{3}$ Hospital Vitarte, Lima, Perú

${ }^{4}$ E-mail: amanchegos@gmail.com

Recibido: 18 de abril de 2016

Aceptado para publicación: 28 de enero de 2017 
This study aimed to determine the effect of L-glutamine on the expression of immunoglobulin A (IgA) and cytokines TGF- $\beta$ and interleukins IL-4, IL-5, IL-6 and IL-10 in the mucosa of the jejunum of 4-7 days old alpacas. A group of 6 alpacas was orally administered $3.3 \mathrm{mM}$ L-glutamine per kg body weight and a second dose 7 days later. A second group of 7 alpacas received PBS as control. All animals were euthanized 3 days after the treatment and a portion of the jejunum was collected. The messenger RNA (mRNA) was extracted and the reverse transcription (RT) and real-time PCR was done using primers specific for each gene in study. The $2^{-\Delta \Delta c t}$ method was used for the relative quantitation of IgA, TGF- $\beta$ and interleukins. The TGF- $\beta$ expressed 3 times more and IgA 18 times more in alpacas treated with L-glutamine than in the controls $(\mathrm{p}<0.05)$. The expressions of IL-4, IL-5, IL-6 and IL-10 showed similar levels in the treated and control animals. It is concluded that L-glutamine increases the expression of TGF- $\beta$ and IgA improving the humoral adaptive immune response of intestinal mucosa in new-born alpacas.

Key words: South American camelids, mucosal immunity, glutamine, interleukins

\section{INTRODUCCIÓN}

A partir del nacimiento, la alpaca debe desarrollar un eficiente sistema inmune para soportar el desafío de microorganismos patógenos que existen en su entorno. El intestino empieza a ser colonizado con microorganismos saprófitos y potencialmente patógenos que forman la microflora intestinal, originando continuas señalizaciones para la respuesta inmune (Pamer, 2007), incluyendo la proliferación de células linfoides diferenciadas para la producción de la expresión de diversas citoquinas e interleucinas que dirigen la respuesta inmune (Kaiserlian et al., 2005). Estas células son altamente degradadoras de glutamina, conteniendo las enzimas necesarias para metabolizarlas; siendo así que se puede apoyar su necesidad energética agregando L-glutamina, como ha sido determinado en ratones y cerdo ( $\mathrm{Li}$ et al., 1997; Kudsk et al., 2000).

La glutamina promueve y mantiene la función de varios órganos y células como el riñón (Conjard et al., 2002), intestino (Ra- mos Lima et al., 2002), hígado (de Souza et al., 2001), corazón (Khogali et al., 2002), linfocitos (Curi et al., 1999), macrófagos (Newsholme et al., 1986) y neutrófilos (Pithon-Curi et al., 2003). Al más básico nivel, la glutamina sirve como un combustible importante en estas células y tejidos. Una tasa alta de absorción de glutamina es característica de las células de división rápida como los enterocitos, fibroblastos y linfocitos (Curi et al., 1999), donde la glutamina es un precursor importante de péptidos y proteínas, así como de aminoazúcares, purinas y pirimidinas, al participar en la síntesis de nucleótidos y ácidos nucleicos (Szondy y Newsholme, 1990; Boza et al., 2000).

La glutamina, al ser un precursor esencial para la síntesis de purinas y pirimidinas, es requerida para la proliferación de linfocitos. El incremento de concentraciones extracelulares de glutamina de 0.01 a $0.5 \mathrm{mM}$ (nivel fisiológico en plasma) incrementa la proliferación linfocitaria (Wu et al., 1992). Asimismo, la arginina derivada de glutamina parece ser esencial para la actividad de los macrófagos (Murphy y Newsholme, 1998). 
El tejido linfoide asociado al intestino (GALT, gut-associated lymphoid tissue) es un componente esencial de la defensa intestinal. Diversos estudios han demostrado que no solo la ingesta proteica total, sino la disponibilidad de aminoácidos dietéticos específicos, particularmente de glutamina, glutamato, arginina, metionina, cisteína y treonina son esenciales para la optimización de la función inmune del intestino y de las células inmunes residentes próximas (Li et al., 2007). Estos aminoácidos promueven el mantenimiento de la integridad, crecimiento y función intestinal, así como también, la normalización de la secreción de citoquinas inflamatorias y refuerzan el número de linfocitos $\mathrm{T}$, funciones específicas de células $\mathrm{T}$ y la secreción de IgA por las células de la lámina propia (Ruth y Field, 2013).

Los mecanismos protectores de la glutamina (Gln) a nivel intestinal pueden estar asociados al incremento de la expresión de proteínas de shock térmico de $70 \mathrm{KDa}$ (70 kilodalton heat shock proteins - Hsp70) (Zhong et al., 2011). Asimismo, se ha observado una mitigación en el crecimiento de $E$. coli $\mathrm{K} 88+$ en cerdos desafiados, principalmente debido al mantenimiento de la integridad de la función y morfología intestinal (Yi et al., 2005). Wang et al. (2008) demostraron que la administración de glutamina en la dieta de lechones reforzó la capacidad de defensa oxidativa intestinal, previno la atrofia yeyunal y promovió el crecimiento intestinal y ganancia de peso.

El presente trabajo tuvo como objetivo establecer el efecto positivo de L-glutamina en el desarrollo de la respuesta inmune humoral de la mucosa intestinal de alpacas de corta edad, proyectando su posible uso como medida protectora de infecciones en la crianza de alpacas.

\section{Materiales y Métodos}

La suplementación de animales con Lglutamina y la toma de muestras intestinales se realizó en la Estación Experimental Maranganí del Centro de Investigación IVITA (Cusco, Perú). El estudio de la cuantificación de citoquinas e interleucinas se realizó en el Laboratorio de Virología de la Facultad de Medicina Veterinaria, Universidad Nacional Mayor de San Marcos, Lima.

\section{Obtención y Procesamiento de Muestras}

Se trabajó con 13 crías de alpaca, aparentemente sanas, de 4 a 7 días de edad y sin distinción de sexo, del IVITA-Maranganí. Seis de ellas fueron dosificados con L-glutamina (grupo A) y las otras siete quedaron como controles no tratados (grupo B).

Se estudió la expresión de citoquinas que regulan positivamente la producción de IgA en la mucosa intestinal (TGF- $\beta$, IL-4, IL5, IL-6 e IL-10), y se determinó la inmunoglobulina A. El grupo A tratado, recibió una dosis de L-glutamina (G7513 SIGMA-Aldrich) en la concentración de $3.3 \mathrm{mM} / \mathrm{kg}$ de peso vivo en $5 \mathrm{ml}$ de PBS al inicio del estudio y una dosis adicional a los 7 días. El grupo $\mathrm{B}$ control solo recibió $5 \mathrm{ml}$ de PBS en las mismas fechas. La concentración de L glutamina utilizada fue seleccionada considerando que las concentraciones típicas en medios empleados para la fabricación biológica y la aplicación de ingeniería tisular de encuentra entre 2 y 4 mM (Heeneman et al., 1993)

Los animales fueron manejados bajo las normas bioéticas del centro de investigación, bajo el Protocolo de Autorización N. ${ }^{\circ}$ 2009001 del Comité de Ética y Bienestar Animal de la Facultad de Medicina Veterinaria de la Universidad Nacional Mayor de San Marcos. Para el sacrificio, tres días después de la segunda dosificación, las alpacas fueron sedadas con xilacina-ketamina $(7.5 \mathrm{mg} / \mathrm{kg}$, vía i.m.) y la eutanasia se hizo con sobredosis de pentobarbital sódico.

Se tomaron tres porciones de $2 \mathrm{~cm}$ de longitud del segmento medio del yeyuno de forma aséptica (el yeyuno contiene el mayor número de linfocitos intraepiteliales). Las 
muestras fueron inmediatamente lavadas en suero fisiológico al $0.9 \%$ estéril para eliminar restos de contenido intestinal. Se colocaron en crioviales estériles de $2 \mathrm{ml}$ de capacidad y se congelaron en nitrógeno líquido para su transporte hacia el laboratorio.

Las muestras intestinales fueron descongeladas gradualmente desde $-196^{\circ} \mathrm{C}$ seguido de $-70^{\circ} \mathrm{C},-20^{\circ} \mathrm{C}$ y a $+4{ }^{\circ} \mathrm{C}$ en intervalo de tres horas por cada rango de temperatura. Se hizo un raspado profundo del tejido intestinal con hoja de bisturí a $4{ }^{\circ} \mathrm{C}$ sobre placa Petri estéril. El material del raspado fue lavado dos veces en PBS $0.15 \mathrm{M}$ pH $7.2 \mathrm{y}$ centrifugado a $3000 \mathrm{~g}$ por minuto después de cada lavado para eliminar posibles restos de contenido intestinal inhibidores del PCR. El pellet final fue resuspendido en $500 \mu 1$ de agua libre de nucleasas y congelado a $-70^{\circ} \mathrm{C}$ hasta la extracción de ARN total.

\section{Extracción de ARN Total}

Para la extracción del ARN se empleó TRIzol ${ }^{\circledR}$ (Invitrogen, EEUU) siguiendo las instrucciones del fabricante. Brevemente, las muestras fueron incubadas a temperatura ambiente por $3 \mathrm{~min}$ con el reactivo y luego se adicionó cloroformo frío. Se centrifugó, se tomó la fase acuosa de la mezcla y el ARN total se precipitó con alcohol isopropílico frío. Se centrifugó y lavó con etanol frío al 70\%. Finalmente, el ARN se resuspendió en $60 \mu 1$ de agua libre de nucleasas y almacenado a $70{ }^{\circ} \mathrm{C}$ hasta su uso en el RT-PCR.

\section{Síntesis de ADN Complementario por (ADNc) Transcripción Inversa (RT)}

El ARN de cada muestra fue tomado como templado para la síntesis de ADNc empleando el kit SuperScript $₫$ III First-Strand Synthesis SuperMix for qRT-PCR (Invitrogen, EEUU), siguiendo las instrucciones del fabricante. El kit emplea la transcriptasa reversa MuLV H+ para generar ADNc a partir de moléculas de ARN templado en las muestras y viene proveído de dNTP, hexámeros al azar
(0.2 $\mu \mathrm{M}$, concentración final) y sales para optimizar la reacción.

Las concentraciones finales de los componentes de la síntesis de ADNc se colocaron en un volumen final de $20 \mu 1$ por reacción/muestra. Se añadió $2 \mu 1$ de ARN templado de cada una de las muestras en los tubos correspondientes de modo que el templado no exceda el $10 \%$ del volumen final de reacción. Las muestras se llevaron al termociclador PTC-200 Engine Chromo IV (MJ Research-Biorad, EEUU) programado con el siguiente protocolo: $25^{\circ} \mathrm{C}$ por $10 \mathrm{~min}$, $50{ }^{\circ} \mathrm{C}$ por $30 \mathrm{~min}, 85^{\circ} \mathrm{C}$ por $5 \mathrm{~min}$. Se añadió 2 UI de RNasa $\mathrm{H}+$ de $E$. coli (provisto por el kit) a cada pocillo y se incubó a $37^{\circ} \mathrm{C}$ por 20 min para destruir la molécula ARN del híbrido ARN: ADNc. El ADNc obtenido fue congelado a $-70{ }^{\circ} \mathrm{C}$ hasta su uso en la reacción de PCR Tiempo Real.

\section{PCR Tiempo Real}

En el PCR Tiempo Real se usaron los oligonucleótidos correspondientes a la citoquina TGF- $\beta$, las interleucinas $4,5,6$ y 10 e IgA (Cuadro 1). Se incluyeron oligonucleótidos para la amplificación específica de transcritos de GAPDH como gen interno para la normalización de la cuantificación relativa.

Se emplearon $2 \mu 1$ del ADNc de cada una de las muestras como templado para la reacción de PCR con el kit SuperScript ${ }^{\circledR}$ III First-Strand Synthesis SuperMix for qRTPCR (Invitrogen, EEUU), siguiendo las recomendaciones del fabricante. La reacción PCR utiliza la polimerasa Hot Start Taq Platinum modificada para mayor estabilidad contenida en $2 x$ qPCR master mix que contiene SYBR Green II, buffer, $5 \mathrm{mM}$ de $\mathrm{MgCl}_{2}$, dNTP mix $(0.2 \mu \mathrm{M}$ concentración final $) \mathrm{y}$ como referencia pasiva se utilizó ROX, provisto por el kit.

Los oligonucleótidos fueron diluidos en agua libre de nucleasas para obtener soluciones de trabajo de $10 \mu \mathrm{M}$ de forma que su con- 
Cuadro 1. Cebadores utilizados en el estudio

\begin{tabular}{llll}
\hline Gen & Cebador hacia adelante & Cebador Reverso & $\begin{array}{l}\text { Secuencia } \\
\text { genbank }\end{array}$ \\
\hline GAPDH & ATCACTGCCACCCAGAAGAC & GCACGTCAGATCCACAACAG & XM_006210852.1 \\
IL-4 & TCATCAAAACGCTGAACACC & CGCTGAGGTTCCTGCAAGT & XM_006212827.1 \\
IL-5 & GATAGGCGAYGGGAACTTGA & GCCATCTTYCTCCTCCACAC & XM_006212831.1 \\
IL-6 & CCTGGTGATGGCTACTGCTT & ACAGTGCCTCCTTGCTGTTT & XM_006201793.1 \\
IL10 & TTACCTGGAGGAGGTGATGC & GGCTTTGTAGACCCCCTTCT & XM_006215461.1 \\
TGF $\beta$ & GAGGTGATCTYGCCACCATT & GTCCTTGCGGAAGTCAATGT & NM_001290071.1 \\
IgA Exon3 & AAGGACGTGCTGGTTCGAT & CCCACCATGCAGGAGTAGTT & AM773729.1 \\
\hline
\end{tabular}

Cuadro 2. Cuantificación relativa de la expresión de los genes en estudio en la mucosa yeyunal de crías de alpaca tratadas con L-glutamina

\begin{tabular}{lcccccc}
\hline Grupo & $\begin{array}{c}\text { IL-4 } \\
(\mathrm{x} \pm d e)\end{array}$ & $\begin{array}{c}\text { IL-5 } \\
(\mathrm{x} \pm d e)\end{array}$ & $\begin{array}{c}\text { IL-6 } \\
(\mathrm{x} \pm d e)\end{array}$ & $\begin{array}{c}\text { IL-10 } \\
(\mathrm{x} \pm d e)\end{array}$ & $\begin{array}{c}\text { TGF } \beta \\
(\mathrm{x} \pm d e)\end{array}$ & $\begin{array}{c}\text { IgA } \\
(\mathrm{x} \pm d e)\end{array}$ \\
\hline Control & $7.7 \pm 4.0^{\mathrm{a}}$ & $1.1 \pm 1.1^{\mathrm{a}}$ & $1.2 \pm 1.2^{\mathrm{a}}$ & $0.1 \pm 0.1^{\mathrm{a}}$ & $0.6 \pm 0.5^{\mathrm{a}}$ & $3.5 \pm 4.6^{\mathrm{a}}$ \\
Tratados & $7.3 \pm 5.0^{\mathrm{a}}$ & $1.4 \pm 0.9^{\mathrm{a}}$ & $6.2 \pm 13.3^{\mathrm{a}}$ & $0.2 \pm 0.2^{\mathrm{a}}$ & $1.8 \pm 0.9^{\mathrm{b}}$ & $46.2 \pm 37.5^{\mathrm{b}}$ \\
\hline
\end{tabular}

a,b Superíndices diferentes indican significancia estadística $(p<0.05)$

centración final en la reacción fue de $0.2 \mu \mathrm{M}$. Se utilizó el termociclador PTC 200 (Peltier Therme Cycler) Chromo 4 (detector continuo de fluorescencia) de MJ ResearchBioRad (EEUU), programado según el set de oligonucleótidos empleado: $50^{\circ} \mathrm{C}$ por $2 \mathrm{~min}$, $95^{\circ} \mathrm{C}$ por 10 min y 45 ciclos de $95^{\circ} \mathrm{C}$ por $15 \mathrm{~s}$, $60{ }^{\circ} \mathrm{C}$ por $60 \mathrm{~s}$. Temperatura de disociación de $65-95{ }^{\circ} \mathrm{C}$ cada $0.3{ }^{\circ} \mathrm{C}, 2 \mathrm{~s}$. El software empleado para el termociclador y el cabezal Chromo 4 fue el Opticon Monitor 2 v. 2.0.3.

Adicionalmente, las mezclas de los productos fueron sometidos a electroforesis en gel de agarosa al 1.5\% teñidos con bromuro de etidio y visualizados en un Transiluminador UV (UltraLum, EEUU) para comprobar su pureza e identidad a través de la comprobación de su longitud en pares base. Los resultados fueron observados y analizados utilizando el programa Opticon Monitor 2.1 (MJ Research-BioRad, EEUU).

\section{Cuantificación Relativa y Análisis Esta- dístico}

Se empleó la técnica $2^{-\Delta \Delta C t}$ como técnica de cuantificación de la expresión relativa de genes detectables a través de RT-PCR Tiempo Real (Livak y Smittdgen, 2001). El método se basa en el análisis comparativo de las curvas de amplificación $(\mathrm{Ct})$ de las muestras a estudiar con el del calibrador (fetos de 11 meses de gestación), comparados con el 
Ct de un control endógeno (GAPDH). Los resultados se interpretan indicando que el gen se ha expresado $\langle\langle x\rangle$ veces con respecto al calibrador.

Se empleó la prueba de Shapiro-Wilk para saber si las variables siguen la distribución normal. Las que siguieron la distribución normal se trabajaron con «t $»$ Student (IgA y TGF- $\beta$ ) y las otras (IL4, IL5, IL6 e IL10) con la prueba no paramétrica U de Mann Whitney-Wilcoxon para el análisis de significancia entre los grupos tratados y control.

\section{Resultados}

Los resultados obtenidos en los animales no tratados demuestran que en todas las crías de alpacas hubo expresión de la citosina e interleucinas relacionadas a la inmunidad adquirida humoral de la inmunoglobulina Aque expresan el exón 3 en su mucosa intestinal (Cuadro 2).

El análisis molecular de la expresión de las interleucinas IL-4, IL-5, IL6 e IL-10 demostró que no existe diferencia significativa entre las crías de alpacas controles y las tratadas con L-glutamina (Cuadro 2). La expresión de la citocina TGF $\beta$ en las crías tratadas fue tres veces mayor que en las no tratadas $(\mathrm{p}<0.05)$. Asimismo, la expresión de IL6 fue 6.2 veces mayor que el calibrador (feto a término) en las crías tratadas con Lglutamina que en los controles (1.2 veces); sin embargo, estas diferencias no fueron estadísticamente significativas por el amplio rango de variabilidad obtenido en los animales tratados (Cuadro 2).

En forma similar, el gen de IgA en los animales tratados se expresó 46.2 veces más que el calibrador, mientras que los animales no tratados expresaron solo 3.5 veces más que el calibrador, indicando una diferencia significativa ( $<<0.05$; Cuadro 2$)$.

\section{Discusión}

No se disponen de trabajos similares en alpacas, pero existen estudios en la suplementación de glutamina y glutamato en conejo (Chamorro et al., 2009) y cerdo (Wang et al., 2008; Wu et al., 2011) donde se evalúan parámetros productivos y de protección contra enfermedades. En el presente estudio, las alpacas recibieron L-glutamina en concentración de $3.3 \mathrm{mM} / \mathrm{kg}$, vía oral, pero a diferencia de los otros estudios, solo se le suministró dos veces y con intervalo de 7 días para medir molecularmente los cambios en la expresión de las citoquinas que inducen a una mayor producción de IgA en la mucosa intestinal. La concentración utilizada es mayor a la concentración de $0.7 \mathrm{mM}$ en el plasma sanguíneo del humano y está dentro del rango de una concentración de glutamina intracelular (2-20 mM) (Newsholme et al., 2003). Se estima que toda la L-glutamina suplementada sea metabolizada en la mucosa intestinal, ya que este tejido es considerado consumidor de glutamina (Ramos Lima et al., 2002).

Si bien es cierto la glutamina es suplementada naturalmente por la leche en las especies mamíferas, en donde tiene mayores concentraciones junto a prolina, metionina, isoleucina y lisina (Davis et al., 1994), las necesidades de este aminoácido son altas durante el estado neonatal por el crecimiento del intestino y otros órganos que lo requieren, de allí que los suplementos alimenticios con glutamina mejoran el crecimiento de las crías lactantes (Wang et al., 2008). En el estudio, la administración de L-glutamina busca proporcionar su disponibilidad inmediata durante la estimulación de la respuesta inmune de la mucosa intestinal, lo cual se manifiesta con la elevación de la producción de $\operatorname{IgA}$ en el intestino; similar a lo que ocurre en ratones suplementados con glutamina en la etapa neonatal (Yu-Ni et al., 2004). 
Fukatsu et al. (2001) determinaron en ratones que los niveles de expresión de las interleucinas IL-4 e IL-10, así como de IgA no se modificaron al ser suplementados con $2 \%$ de L-glutamina. En el presente estudio, no se elevaron los niveles de expresión de IL4 e IL10 en comparación con la respuesta de los animales controles debido a que, tal vez, ya se encontraban expresando los niveles adecuados de estas interleucinas, si se considera que sus expresiones son negativamente reguladas por la presencia de interleucinas que promueven la inmunidad de tipo células con un perfil Th1. Está demostrado que en la mucosa intestinal de las crías de alpacas predominan la expresión de las interleucinas de este perfil como el Interferon gamma (IFN $\gamma$ ) (Chiok, 2012).

El incremento de IgA en las crías de alpaca tratadas con L-glutamina sin incremento de IL-4 e IL-10 es controversial, ya que estas dos interleucinas participan en la diferenciación y activación de los linfocitos $\mathrm{B}$ en células plasmáticas productoras de IgA junto con el Factor de Crecimiento Transformante â (TGF- $\beta$ ) en la mucosa intestinal (Fagarasan y Honjo, 2003), el cual si se eleva tres veces más que en los animales no suplementados. La función de TGF- $\beta$ parece ser clave para el incremento de IgA en este estudio, ya que interviene en procesos inmunes $\mathrm{e}$ inflamatorios, pues suprime el crecimiento y diferenciación de muchos linajes de células inmunes, incluyendo células T y B. El TGF- $\beta$ es producido por todas y cada una de las células de linaje inmunológico y actúa de una manera autocrina y paracrina. Además de la regulación de la proliferación de células del sistema de defensa, regula la expresión de moléculas de adhesión, especialmente en la médula ósea y en el microambiente tímico (Sporn, 1999). Asimismo, las células T que entran en apoptosis normal son el mayor objetivo del TGF- $\beta$, con una acción inmunosupresora drástica, e in vivo aumenta las funciones efectoras y de memoria de los linfocitos T CD4+ antígeno-específicos, inhibe la secreción de IgG e IgM, controla la proliferación y maduración en células $\mathrm{B}$ y tie- ne un papel regulatorio crítico en la expresión de IgA (Moustakas et al., 2002). En el presente estudio pareciera que ayuda a incrementar la expresión del gen de la IgA, que incluye el exón 3.

Teniendo en cuenta que la L-glutamina es un aminoácido que participa en varios procesos específicos celulares, incluyendo el metabolismo (combustible oxidativo, precursor de la gluconeogénesis, y precursor lipogénico), la integridad celular (apoptosis, la proliferación celular), la síntesis y degradación de proteínas, potencial redox, estallido respiratorio (Curi et al., 2005), debe mejorar la producción de IgA de células plasmáticas ya diferenciadas. Esto quedó demostrado al expresarse 18 veces más en comparación a los controles ( $\sin$ aumento de la expresión de IL-4 e IL-10).

La IL-6 se encontró ligeramente elevada en las crías tratadas con L-glutamina. Esta interleucina está asociada a procesos inflamatorios; sin embargo, se ha determinado que los enterocitos y colocitos producen IL-6 en intestino delgado y grueso, haya o no un proceso inflamatorio de por medio, por lo que es necesario mayor investigación en su desempeño en el papel activo en la respuesta inmune del intestino (Jones et al., 1993). La L-glutamina eleva el estallido oxidativo de macrófagos y fagocitos (Wu et al., 2004), lo cual podría incrementar la actividad de estas células e incrementar la producción de citoquinas proinflamatorias, como IL-6.

La ausencia de respuesta de las interleucinas IL4, IL5 e IL10 pudo deberse al bajo estímulo de la L-glutamina por la dosis empleada, ya que en un ensayo en cerdos donde fue suplementada en forma diaria, demostró su efecto benéfico en la protección contra infecciones de $E$ coli (Yi et al., 2005).

\section{Agradecimientos}

Los autores manifiestan su agradecimiento a INNOVATE PERU por el financiamiento del trabajo dentro del marco del proyecto FINCyT N. ${ }^{\circ} 180-2013$. 


\section{Literatura Citada}

1. Boza JJ, Moёnnoz, D, Bournot CE, Blum S, Zbinden I, Finot PA, Ballèvre O. 2000. Role of glutamine on the novo purine nucleotide synthesis in Caco-2 cells. Eur J Nutr 39: 38-46. doi: 10.1007/ s003940050074

2. Chamorro S, de Blas C, Grant G, Badiola I, Menoyo D, Carabaño R. 2009. Effect of dietary supplementation with glutamine and a combination of glutamine-arginine on intestinal health in twenty-five-day-old weaned rabbits. J Anim Sci 88: 170-180. doi: 10.2527/ jas.2008-1698

3. Chiok K. 2012. Expresión de citoquinas de la respuesta Th1 (IFN- $\gamma$, IL-2) y Th2 (IL-4, IL-10) en mucosa intestinal de crías de alpaca (Vicugna pacos) sanas y con enteropatía. Tesis de Magíster. Lima: Univ Nacional Mayor de San Marcos. 88 p.

4. Conjard A, Brun V, Martin M, Baverei G, Ferrier B. 2002. Effect of starvation on glutamine ammoniagenesis and gluconeogenesis in isolated mouse kidney tubules. Biochem J 368: 301-308. doi: 10.1042/BJ20020331

5. Curi R, Newsholme P, Pithon-Curi TC, Pires-de-Melo M, Garcia C, et al. 1999. Metabolic fate of glutamine in lymphocytes, macrophages and neutrophils. Braz J Med Biol Res 32: 15-21. doi: 10.1590/S0100-879X1999000100002

6. Curi R, Lagranha C, Doi S, Sellitti D, Procopio J, Pithon-Curi T, Corless M, Newsholme P. 2005. Molecular mechanisms of glutamine action. J Cell Physiol 204: 392-401. doi: 10.1002/jcp.2033

7. Davis TA, Nguyen HV, Garcia-Bravo R, Fiorotto ML, Jackson EM, Reeds PJ. 1994. Amino acid composition of the milk of some mammalian species changes with stage of lactation. Brit $\mathrm{J}$ Nutr 72: 845-853. doi: 10.1079/ BJN19940089
8. De Souza HM, Borba-Murad GR, Ceddia RB, Curi R, VardanegaPeicher M, Bazotte RB. 2001. Rat liver responsiveness to gluconeogenic substrates during insulin-induced hypoglycemia. Braz J Med Biol Res 34: 771-777. doi: 10.1590/S0100879X2001000600012

9. Fagarasan S, Honjo T. 2003. Intestinal IgA synthesis: regulation of front-line body defenses. Nat Rev Immunol 3: 6372. doi: $10.1038 /$ nri982

10. Fukatsu K, Kudsk KA, Zarzaur BL, Wu Y, Hanna MK, DeWitt RC. 2001. TPN decreases IL-4 and IL-10 mRNA expression in lipopolysaccharide stimulated intestinal lamina propria cells but glutamine supplementation preserves the expression. Shock 15:318-322.

11. Heeneman S, Deutz NE, Buurman $W A$. 1993. The concentrations of glutamine and ammonia in commercially available cell culture media. J Immunol Methods 166: 85-91. doi: 10.1016/00221759(93)90331-Z

12. Jones SC, Trejdosiewicz LK, Banks RE, Howdle PD, Axon AT, Dixon MF, Whicher JT. 1993. Expression of interleukin- 6 by intestinal enterocytes. $\mathrm{J}$ Clin Pathol 46: 1097-1100.

13. Kaiserlian D, Cerf-Bensussan N, Hosmalin A. 2005. The mucosal immune system: from control of inflammation to protection against infections. J Leukoc Biol 78: 311-318. doi: 10.1189/jlb.0105053

14. Khogali SE, Pringle SD, Weryk BV. Rennie MJ. 2002. Is glutamine beneficial in ischemic heart disease? Nutrition 18: 123-126. doi: 10.1016/ S0899-9007(01)00768-7

15. Kudsk KA, Wu Y, Fukatsu K, Zarzaur BL, Johnson CD, Wang R, Hanna MK. 2000. Glutamine-enriched total parenteral nutrition maintains intestinal interleukin-4 and mucosal immunoglobulin A levels. JPEN J Parenter Enteral Nutr 24: 270-274. 
16. Li J, Kudsk KA, Janu P, Renegar KB. 1997. Effect of glutamine-enriched total parenteral nutrition on small intestinal gut-associated lymphoid tissue and upper respiratory tract immunity. Surgery 121 : 542-549. doi: 10.1016/S0039. 6060(97)90109-4

17. Li P, Yin Y-L, Li D, Woo Kim S, Wu G 2007. Amino acids and immune function. Br J Nutr 98: 237-252. doi: 10.1017/S000711450769936X

18. Livak KJ, Schmittgen TD. 2001. Analysis of relative gene expression data using Real-Time Quantitative PCR and

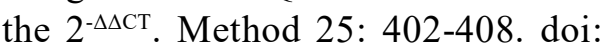
10.1006/meth.2001.1262

19. Moustakas A, Pardali K, Gaal A, Heldin CH. 2002. Mechanism of TGFbeta signaling in regulation of cell growth and differentiation. Immunol Lett 82: 8591. doi: 10.1016/S0165-2478(02)00023-8

20. Murphy C, Newsholme P. 1998. Importance of glutamine metabolism in murine macrophages and human monocytes to L-arginine biosynthesis and rates of nitrite or urea production. Clin Sci (Lond) 95: 397-407. doi: 10.1042/ CS19980194

21. Newsholme P, Curi R, Gordon S, Newsholme EA. 1986. Metabolism of glucose, glutamine, long-chain fatty acids and ketone bodies by murine macrophages. Biochem J 239: 121-125.

22. Newsholme P, Procopio J, Lima MM, Pithon-Curi TC, Curi R. 2003. Glutamine and glutamate-their central role in cell metabolism and function. Cell Biochem Funct 21: 1-9. doi: 10.1002/ cbf. 1003

23. Pamer E. 2007. Immune responses to commensal and environmental microbes. Nat Immunol 8: 1173-1178. doi: 10.1038/ ni1526

24. Pithon-Curi TC, Schumacher RI, Freitas JJ, Lagranha C, Newsholme P, Palanch AC, Doi SQ, Curi R. 2003. Glutamine delays spontaneous apoptosis in neutrophils. Am J Physiol Cell Physiol
284: C1355- C1361 doi: 10.1152/ ajpcell.00224.2002

25. Ramos Lima MM, de Mello MA, Curi R. 2002. Walker 256 tumour growth causes marked changes of glutamine metabolism in rat small intestine. Cell Biochem Funct 20: 107-113. doi: 10.1002/ cbf.961

26. Ruth M, Field C. 2013. The immune modifying effects of amino acids on gutassociated lymphoid tissue. J Anim Sci Biotechnol4: 27. doi: 10.1186/2049-18914-27

27. Sporn M. 1999. TGF- $\beta$ : 20 years and counting. Microbes Infect 1: 1251-1253.

28. Szondy Z, Newsholme EA. 1990. The effect of various concentrations of nucleobases, nucleosides or glutamine on the incorporation of $[3 \mathrm{H}]$ thymidine into DNA in rat mesenteric-lymph-node lymphocytes stimulated by phytohaemagglutinin. Biochem J 270: 437-440.

29. Wang J, Chen L, Li P, Li X, Zhou H, Wang F, Li D, et al. 2008. Gene expression is altered in piglet small intestine by weaning and dietary glutamine supplementation. J Nutr 138: 1025-1032.

30. Wu G, Field CJ, Marliss EB. 1992. Enhanced glutamine and glucose metabolism in cultured rats splenocytes stimulated by phorbol myristate acetate plus ionomycin. Metabolism 41:982-988.

31. Wu G, Fang YZ, Yang S, Lupton JR, Turner ND. 2004. Glutathione metabolism and its implications for health. J Nutr 134: 489-492.

32. Wu G, Bazer F, Johnson G, Knabe D, Burghardt R, Spencer T, Li X, Wang $J$. 2011. Triennial growth symphosium: important roles for L-glutamine in swine nutrition and production. J Anim Sci 89: 2017-2030. doi: 10.2527/jas.2010-3614

33. Yi G, Carroll J, Allee G, Gaines A, Kendall D, Usry J, Toride Y, Izuru S. 2005. Effect of glutamine and spraydried plasma on growth performance, small intestinal morphology and immune responses of Escherichia coli K88+- 
challenged weaned pigs. J Anim Sci 83: 634-643.

34. Yu-Ni L, Sung-Ling Y, Ming-Tsan L, Huey-Fang S, Chiu-Li Y, Wei-Jao C. 2004. Glutamine supplementation enhances mucosal immunity in rats with gut-derived sepsis. Nutrition 20: 286-291. doi: 10.1016/j.nut.2003.11.015
35. Zhong X, Zhang X, Li M, Zhou Y, Li W, Huang X, Zhang L, Wang T. 2011. Intestinal growth and morphology is associated with the increase in heat shock protein 70 expression in weaning piglets through supplementation with glutamine. J Anim Sci 89: 3634-3642. doi: 10.2527/jas.2010-3751 